\title{
Antiretroviral therapy and HIV-associated cancers: Anti- angiogenic effect of efavirenz on chick chorioallantoic membrane
}

\author{
Rosie T McNeil ${ }^{1 *}$, Clement B Penny ${ }^{2}$ and Margot $\mathrm{J} \mathrm{Hosie}^{3}$ \\ ${ }^{1}$ Anatomy Department, Sefako Makgatho Health Sciences University (SMU), Ga-Rankuwa, ${ }^{2}$ Department of Internal Medicine, \\ ${ }^{3}$ School of Anatomical Sciences, Medical School, University of the Witwatersrand, Johannesburg, South Africa \\ *For correspondence: Email: rosie.mcneil@smu.ac.za; Tel: +27 719759167
}

Sent for review: 10 January 2016

Revised accepted: 5 October 2017

\begin{abstract}
Purpose: To investigate the anti-angiogenic effect of efavirenz in chick chorioallantoic membrane (CAM).

Methods: Fertile eggs of the domestic fowl (Gallus gallus, variant Domesticus) were used as the in vivo vascular test environment. Experimental groups were VEGF, thalidomide (negative control), efavirenz (test drug), control 1 (treated with diluent) and control 2 (no treatment). CAM photographs were taken using a microscope camera. CAM blood vessels were scored as either "present" or "absent".

Results: There was association between treatment drugs and growth of CAM blood vessels $(p<0.05)$ as follows: control 1-100\%, control 2-67\%, VEGF - $83 \%$, thalidomide - $17 \%$, and efavirenz - $0 \%$ $(n=30)$. Although efavirenz had absolute anti-angiogenicity and presented as a potential anti-cancer drug, there was no significant difference between the anti-angiogenicity of efavirenz (100\%) and thalidomide (83\%) ( $F=1.000, p=0.341)$.

Conclusion: Being a first-line drug in both HAART and combination treatment of HIV-1, efavirenz may be responsible for spontaneous resolution of secondary cancers in HIV-infected patients on HAART regimen.
\end{abstract}

Keywords: Efavirenz, HIV, Cancers, Angiogenesis, Chick chorioallantoic membrane

\begin{abstract}
Tropical Journal of Pharmaceutical Research is indexed by Science Citation Index (SciSearch), Scopus, International Pharmaceutical Abstract, Chemical Abstracts, Embase, Index Copernicus, EBSCO, African Index Medicus, JournalSeek, Journal Citation Reports/Science Edition, Directory of Open Access Journals (DOAJ), African Journal Online, Bioline International, Open-J-Gate and Pharmacy Abstracts
\end{abstract}

\section{INTRODUCTION}

Kaposi sarcoma is the most common cancer seen in patients with acquired immunodeficiency syndrome (AIDS). Many researchers have documented the regression of HIV-associated cancers after antiretroviral therapy (ART) administration [1-5]. This has been particularly reported for lung cancers [6] in relation to the use of "highly active antiretroviral therapy" (HAART). HAART is a drug schedule that involves the use of Efavirenz [7]. HAART induces tumor regression [8] and increases the survival rate among HIV patients with pulmonary Kaposi sarcoma [9]. HAART regime has therefore changed the natural history of HIV/AIDS-related cancers [8]. Moreover, it lengthens the time from onset of treatment to treatment failure [10]. Efavirenz is the most widely used non-nucleoside reverse transcriptase inhibitor (NNRTI) [11].

Vascular endothelial growth factor (VEGF) is a specific and potent mitogen for endothelial cells and its roles in development and pathological angiogenesis have been documented $[12,13]$. It promotes cancer proliferation and metastatic spread by stimulating growth of new vascular networks [14]. The current interest in thalidomide 
lies in its anti-angiogenic properties [15-17]; it antagonizes angiogenic factors such as VEGF [18] and Tumor necrosis factor alpha (TNF-a) $[19,20]$. Efavirenz is the most widely used NNRTI, and is a first line drug in the management of HIV in both adults and children. In combination with other anti-retroviral drugs [21] it suppresses viral load, thereby increasing CD4 count. Nevertheless, Efavirenz has not been adequately studied, even in patients with advanced HIV disease (patients with CD4 counts $<50$ cells $/ \mathrm{mm}^{3)}$ [22].

\section{EXPERIMENTAL}

\section{Ethics approval}

The work was carried out at the University of the Witwatersrand Medical School, under Ethics clearance certificate no. $2008 / 7 / 1$, and also followed the guidelines in the NIH publication no. 85-23, revised 1985 ("Principles of Laboratory Animal Care") [23].

\section{Animals}

Thirty (30) freshly laid, fertile eggs of the New Hampshire breed of chicken (Gallus gallus variant domesticus) and of the same clutches were incubated on the $3^{\text {rd }}$ day of development (Hamburger and Hamilton's stage 21) and used as in vivo systems, a vascular test environment to study angiogenesis. The embryos were staged using the Hamburger and Hamilton staging for chick embryos [24]. Effect of the known angiogenic drug, VEGF, the anti-angiogenic drug, thalidomide, and Efavirenz (anti-retroviral drug) were studied.

\section{Drug administration}

Reagents were purchased sterile and equipment used were autoclaved. Treatments for all groups were given at the same time, on day 3 of incubation as follows: The blunt side of the eggs were sterilized using cotton wool soaked in $70 \%$ ethanol and the respective drugs were administered into the air cells through a single injection, using needles and syringes. The control groups were as follows: VEGF-treated group served as the Positive control group. These subjects were treated with human recombinant VEGF protein (R\&D Systems). A stock solution of $1 \mathrm{mg}$ in $2 \mathrm{ml}$ diluent was first prepared and a single dose of $200 \mu \mathrm{g}$ /embryo was given [25]. Thalidomide was used as the negative control drug. A stock solution of 100 $\mathrm{mg} / \mathrm{ml}$ was prepared and a single dose of 20 mg/embryo was given [26 - 27] Control 1 embryos were treated with equal volume of the diluent ( $1 \mathrm{ml}$ of $70 \%$ ethanol). Control 2 Embryos were not manipulated in any way. The test subjects were treated with efavirenz, obtained from Sequoia Research Products (Pangbourne, UK). A stock solution of $3 \mathrm{mg} / \mathrm{ml}$ was first prepared and treatment /embryo was $200 \mu \mathrm{g}$ [10]. Gomez-Sucerquia ascertained that this drug has a purity of $98-100 \%$ and the solutions are stable when tested by high performance liquid chromatography (HPLC). With the exception of Control 2 subjects, an observation window of $1 \mathrm{x}$ $2 \mathrm{~cm}$ dimension was cut into the egg shells on day 5 of treatment. This was done using a sterilized flexible metal saw.

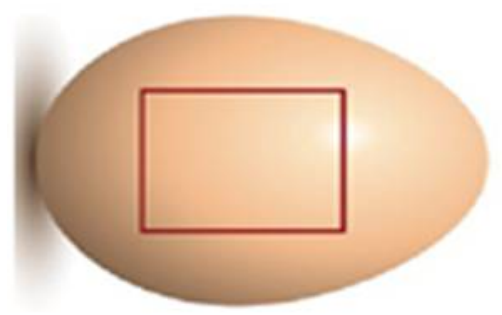

Figure 1: An illustration of the observation window cut in the egg shells

CAM sterility was maintained by instillation of 3 drops of an antibiotic agent ( $50 \mu$ Penstrept in 50 $\mathrm{ml}$ chick ringer) into each egg window. Besides ensuring sterility, this caused the CAM to sink below the egg shell surface, for ease of embryonic CAM observation and photography. CAM images were captured using a Nikon microscope camera. The windows were then sealed with sterilized transparent tapes and the eggs were re-incubated, until day 15 of treatment when CAM images were again taken. Test results were scored based on the presence or absence of blood vessels in embryonic CAMs.

\section{Statistical analysis}

All data were analyzed using Statistical Package for Social Sciences (version 21, SPSS Inc, Chicago, IL) software program. Fisher exact test was used to determine the association between treatment drugs and growth of CAM blood vessels. One-way analysis of variance (ANOVA) was used to compare the anti-angiogenic efficacy of the drugs. $P<0.05$ was considered significant for all analysis.

\section{RESULTS}

\section{Mean angiogenic score}

In Table 1, the results were expressed as mean angiogenic effect $(n=6)$. All control embryos had well-formed CAM blood vessels but the unmanipulated control subjects (control 2), shown 
in Figure 2B and Figure 2D, had a $100 \%$ angiogenicity (mean angiogenic score of 1 ). This was the highest embryonic viability in the experiment. VEGF treatment group (Figure 3) had the next high mean angiogenic score of 0.83 . The manipulated control embryos (Control 1 ) in Figure $2 \mathrm{~A}$ and Figure $2 \mathrm{C}$ had a score of 0.67

Table 1: Fisher's exact test results

\begin{tabular}{lcc}
\hline Treatment drug & $\begin{array}{c}\text { CAM blood } \\
\text { vessel present }\end{array}$ & $\begin{array}{c}\text { CAM blood } \\
\text { vessel absent }\end{array}$ \\
\cline { 2 - 3 } & $\mathrm{f} \%$ & $\mathrm{f} \%$ \\
\hline VEGF & $5(31.3)$ & $1(7.1)$ \\
Thalidomide & $1(6.3)$ & $5(35.7)$ \\
Efavirenz & $0(0.0)$ & $6(42.9)$ \\
Control 1 & $4(25.0)$ & $2(14.3)$ \\
Control 2 & $6(35.5)$ & $0(0.0)$ \\
\hline
\end{tabular}

$P=0.001$ (Fisher's exact test)

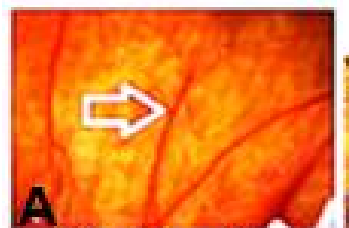

C1: Day 8

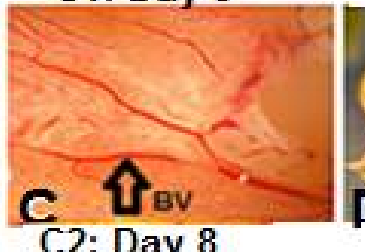

C2: Day 8

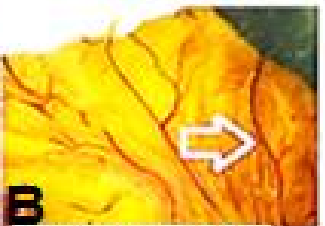

C1: Day 18

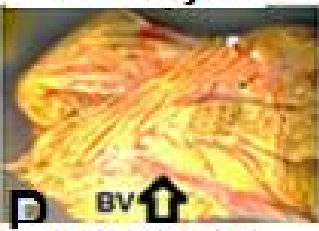

C2: Day 18
Figure 2: Control embryonic CAM. Normal angiogenesis in control embryonic CAMs. C - Control. Control 1 (Figure 2A) and Control 2 (Figure 2B) showed control embryonic CAMs on day 8 of treatment. Arrows point to blood vessels on CAM surface

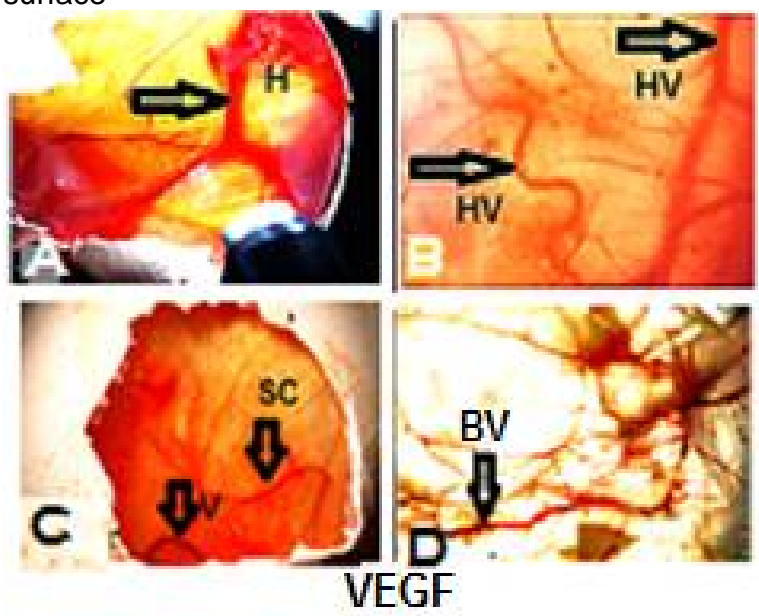

Figure 3: VEGF- treated CAMs. While images A) and $B$ ) were taken on day 8 of incubation (day 5 of treatment); images $\mathrm{C}$ ) and D) were taken on day 18 of incubation (day 15 of treatment). In Figure $3 A$, arrow shows vascular haemorrhage by Day 8 of treatment
There was a significant relationship between drugs used and the growth of CAM blood vessels. Efavirenz treatment group had $42.9 \%$ anti-angiogenicity and $0 \%$ angiogenic effect $(\mathrm{n}=$ $30)$. This was statistically significant $(p=0.001$, Fisher's exact test).

Efavirenz suppressed angiogenesis in all 6 subjects (showing absolute anti-angiogenicity and $0 \%$ angiogenicity). Thalidomide had mean angiogenicity of 0.17 and so, failed as a negative control drug in that embryo.

There was no significant difference between the anti-angiogenic effect of efavirenz and thalidomide. Although efavirenz suppressed both blood vessel development (angiogenesis) and red blood cell formation (erythropoiesis), our work did not measure the anti-erythropoietic effect of treatment drugs.

\section{Morphological features of the embryos}

Figure 2C and Figure 2D show control 1 and control 2 CAMs, respectively, on day 18 of treatment. Both control subjects had established CAM angiogenesis.

Vascular permeability is characteristic of VEGF, which is also known as a permeability factor [15]. In Figure 3B the arrow, HV showed hyper vascularization in VEGF-treated CAM on day 8 of treatment that persisted up to day 15 of treatment (Figure 3C). Note the "small caliber" blood vessels (arrow labelled SC shows). Figure 3D show CAM of an embryo that had died for an unknown reason; its CAM however had wellformed blood (see arrow labelled BV). This incidence seemed peculiar to the embryo.

When compared to control embryos, there were no established vascular network in this treatment group. But patches of erythropoietic activities were found on CAM surfaces on day 8 of treatment, as seen in Figure 4A (labeled E) and figure $4 B$ (labeled $P$ ). By day 15, figure $4 C$ no longer showed angiogenic activity in the CAM but instead, had excessive fibrotic tissue reaction. In Figure 4D, the embryonic CAM had scanty blood islands at the margin of the embryonic CAM (see arrows, labeled M).

Efavirenz suppressed both angiogenesis and erythropoiesis in all the animals. A rather "quiet" CAM was therefore descriptive of this treatment group. The CAM in Figure 5C had exaggerated tissue reaction. In Figure $5 \mathrm{D}$, a thin fibrous structure was seen on the CAM surface. 


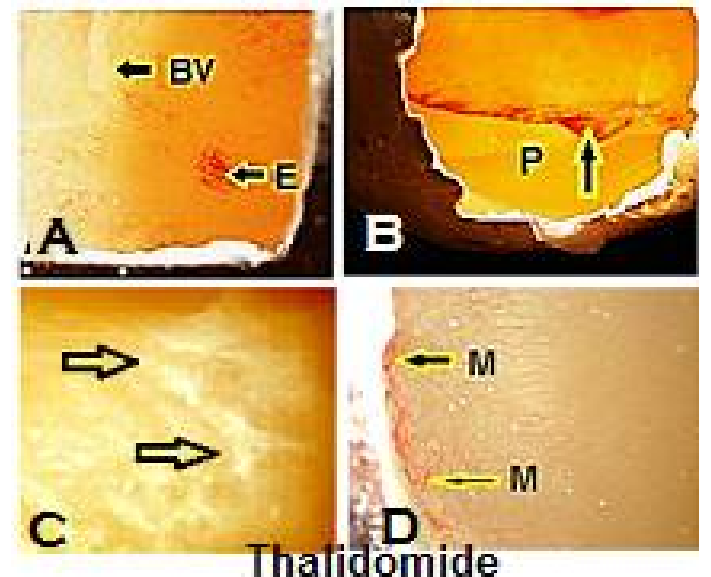

Figure 4: Thalidomide -treated CAMs. While images A) and B) were taken on day 8 of incubation (day 5 of treatment), images $C$ ) and $D$ ) were taken on day 18 of incubation (day 15 of treatment)

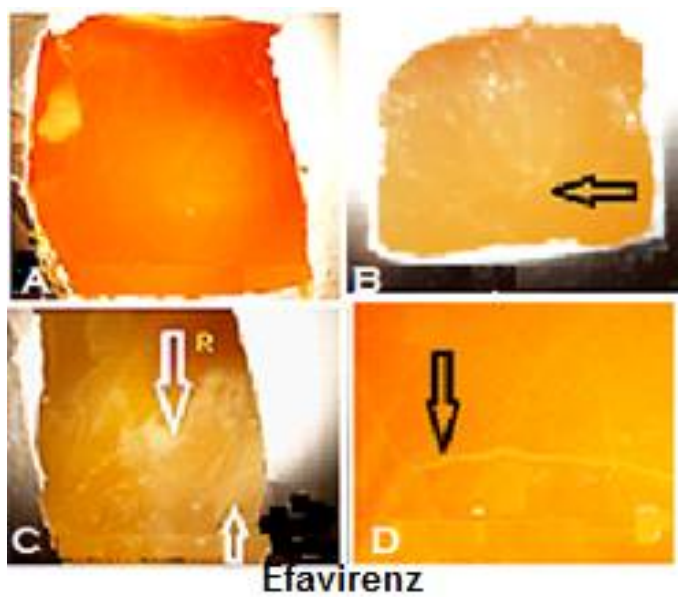

Figure 5: Efavirenz-treated CAMs. While images A) and $B$ ) were taken on day 8 of incubation (day 5 of treatment), images $C$ ) and $D$ ) were taken on day 18 of incubation (day 15 of treatment). No angiogenesis or erythropoiesis seen in any of the animals in this group

\section{DISCUSSION}

Angiogenesis begins with blood vessels forming de novo, from mesenchymal precursor cells called angioblasts. Angioblasts differentiate into endothelial cells that line primordial blood vessels called "blood islands". Blood islands contain progenitor blood cells called haematoblasts [2]. Both blood vessels and blood cells form from embryonic mesoderm. The blood vessels subsequently become organized into a primordial vascular network in the embryo [9].

From the results, the mean angiogenic scores showed that 5 out of 6 VEGF- treated embryos had well established CAM vessels. While vascular growth in the presence of exogenous VEGF surpassed that of Control 1(chick development with embryonic manipulation), it fell short when compared to normal uninterrupted embryonic development (as in Control 2). This suggests that exogenous VEGF did not potentiate the effect of endogenous VEGF in chick CAM. However, treatment with VEGF caused vascular permeability and haemorrhage. This agrees with the fact that VEGF is also known as permeability factor [27]. Vascular exudation causing secondary ascites has occurred in abdominal cancers because cancer cells secreted vascular permeability factor [29]. Furthermore, VEGF treatment caused hyper vascularization in some subjects (Figure $3 \mathrm{~B}$ and $3 \mathrm{C}$. This agrees with the work of Drake et al that high levels of exogenous VEGF caused vascular defects in a way similar to that seen with $50 \%$ reduction in VEGF expression such as in heterozygous deletion of the VEGF allele [28]. Disorder of VEGF expression therefore causes vascular disorganization [4,21]. That is why, although VEGF use has been recommended in the treatment of ischemia and to reduce neurological deficits from stroke, it was found that an initial suppression of VEGF activity is actually necessary to combat vascular permeability and the risk of VEGF-induced hemorrhage [30].

The anti-angiogenic effect of thalidomide treatment failed in one embryo $(n=6)$. Studies of human and animal tumours reveal that occurrence of angiogenesis is crucial in tumorigenesis. Angiogenesis is regulated by both activating and inhibitory growth factors [4]. Growth factors such as VEGF, Tumour necrotic factor (TNF), Epidermal growth factor (EGF), Transforming growth factor (TGF) and Angiogenin play key roles in tumorigenesis $[4,10]$. Anti-angiogenic drugs such as thalidomide suppress cancer growth and enhance patient recovery. It is now known that the resultant amelia in neonates who mothers used thalidomide during pregnancy was due to inhibition of blood vessel growth in the developing fetal limb bud [17]. It has been documented that thalidomide antagonizes angiogenic factors and is thus effective for the treatment of human cancers. From our work we found that although thalidomide -treated CAMs had poor blood vessel network, blood islands with red blood cells were seen at the periphery of CAM surfaces. Unlike thalidomide, the test drug, efavirenz, suppressed both angiogenesis and erythropoiesis in all 6 subjects. This is a significant finding that can be further explored. The $100 \%$ anti-angiogenicity of efavirenz presents it as a potential anti-cancer drug. Distinct CAM pallor therefore characterized this drug treatment group; efavirenz had absolute anti-angiogenic effect and so, $0 \%$ angiogenicity. It contributed $42.9 \%$ anti-angiogenicity to the 
experiment $(n=30, F=9.306, p=0.0005)$. Its anti-angiogenic property surpassed that of thalidomide (35.7 \%). However, when we compared the anti-angiogenicity of Efavirenz to thalidomide (Table 2), there was no significant difference between the anti-angiogenicity of Efavirenz and Thalidomide ( $\mathrm{F}=1.000, p=0.341$, Table 3). This is because our work did not measure the anti-erythropoietic properties of the drugs.

Table 2: Mean angiogenic score

\begin{tabular}{lcc}
\hline Group & $\begin{array}{c}\text { Mean } \\
\text { Angiogenic } \\
\text { effect }\end{array}$ & $\begin{array}{c}\text { Standard } \\
\text { Deviation }\end{array}$ \\
\hline VEGF & 0.83 & 0.41 \\
Thalidomide & 0.17 & 0.41 \\
Efavirenz & 0 & 0 \\
Control 1 & 0.67 & 0.52 \\
Control 2 & 1 & 0 \\
\hline F & &
\end{tabular}

$F=$ 9.306; $p=0.005$

Table 3: Anti-angiogenic effects of efavirenz and thalidomide

\begin{tabular}{llcc}
\hline Drug & $\mathbf{N}$ & Mean & $\begin{array}{c}\text { Standard } \\
\text { deviation }\end{array}$ \\
\hline Thalidomide & 6 & .17 & .41 \\
Efavirenz & 6 & .00 & .00 \\
Total & 12 & .08 & .29 \\
\hline$F=1.000 ; p=0.341$ & & &
\end{tabular}

$F=1.000 ; p=0.341$

We terminated the experiment on day 15 of treatment (day 18 of incubation). At this time, as expected, control CAM blood vessels were still well-formed. One VEGF-treated embryo died for an unknown reason since its CAM blood vessels appeared to be well-formed, 5 subjects in the group were still viable, out of which, 3 embryos had an unusually strong arterial pulsation from hypervascularization.

While one embryo in the thalidomide showed proof of life, 5 in the group and all efavirenztreated subjects had died from lack of oxygenation and nourishment by the end of the experiment. Efavirenz CAM had severe pallor with embryonic resolution. Furthermore, there was CAM tissue thickening. Interruption of angiogenesis disrupts development of epithelial structures [11] and suppresses growth of solid tumours. Studies on inhibitors of the VEGF signaling pathway are currently being done to innovate on cancer treatment [17]. From our findings, there seems to be a promise in the use of efavirenz, beyond that of thalidomide, in the treatment of life-changing conditions such as solid tumors, diabetic retinopathy, and macular degeneration. Although both efavirenz and thalidomide - treated embryos showed signs of inadequate blood supply and nourishment, efavirenz having shown significant antiangiogenic and also anti-erythropoietic properties in chick CAM, has a promising future that that can be exploited in clinical practice.

\section{CONCLUSION}

The findings of this study on efavirenz indicate that it has significant anti-angiogenic effect. Being a first-line drug used in both HAART and combination treatment for HIV infection, the antierythropoietic and anti-angiogenic properties of Efavirenz may be responsible for spontaneous resolution of HIV-associated cancers seen in patients on HAART regimen.

\section{DECLARATIONS}

\section{Acknowledgement}

The authors wish to thank the Health Sciences Faculty of the University of the Witwatersrand for the research grant; Prof JN Maina for providing VEGF protein; staff of the School of Anatomical Sciences (Mrs. Hasiena Ali for her technical support, Donna Paulsen for assisting with the care of the eggs, Mrs. Glynis Veale and Mrs. Alison Mortimer for helping to purchasing chemical reagents); and Prof John Solly for sharing with us air cell injection technique.

\section{Conflict of Interest}

No conflict of interest associated with this work.

\section{Contribution of Authors}

The authors declare that this work was done by the authors named in this article and all liabilities pertaining to claims relating to the content of this article will be borne by them.

\section{Open Access}

This is an Open Access article that uses a funding model which does not charge readers or their institutions for access and distributed under the terms of the Creative Commons Attribution License (http://creativecommons.org/licenses/by/ 4.0) and the Budapest Open Access Initiative (http://www.budapestopenaccessinitiative.org/rea d), which permit unrestricted use, distribution, and reproduction in any medium, provided the original work is properly credited.

\section{REFERENCES}

1. Blum L, Pellet C, Agbalika F, Blanchard G, Morel P, 
Calvo F, Lebbe C. Complete regression of AIDS-related Kaposi's sarcoma associated with undetectable human herpesvirus-8 sequences during anti-HIV protease therapy. AIDS 1997; 11: 1653-1655.

2. Burdick AE, Carmichael C, Rady PL. Tyring SK, Badiavas E. Resolution of Kaposi's sarcoma associated with undetectable level of human herpes virus 8 DNA in a patient with AIDS after protease inhibitor therapy. $J$ Am Acad Dermatol 1997; 37(4): 648-649.

3. Aboulafia DM. Regression of acquired immunodeficiency syndrome-related pulmonary Kaposi's sarcoma after highly active antiretroviral therapy. Mayo Clin Proc 1998; 73(5): 439-443.

4. Benfield T, Kirk O, Elbrond B, Pedersen C. Complete histological regression of Kaposi's sarcoma following treatment with protease inhibitors despite persistence of HHV-8 in lesions. Scand J Infect Dis 1998; 30:613-615.

5. Diz DP, Ocampo HA, Miralles AC, Vázquez GE, Martínez VC. Regression of AIDS-related Kaposi's sarcoma following ritonavir therapy. Oral Oncol 1998; 34(3): 236238.

6. Suneja G, Shiels MS, Melville SK, Williams MA, Rengan $R$, Engels EA, Disparities in the treatment and outcomes of lung cancer among HIV-infected individuals. AIDS 2013; 27(3): 459-468

7. Monini P1, Sgadari C, Toschi E, Barillari G, Ensoli B. Anti-tumour effects of antiretroviral therapy. Nat Rev Cancer 2004; 4: 861-875.

8. Krown SE. Highly Active Antiretroviral Therapy in AIDSAssociated Kaposi's sarcoma: Implications for the Design of Therapeutic Trials in Patients with Advanced, Symptomatic Kaposi's sarcoma. J Clin Oncol 2004, 22(3): 399-402.

9. Holkova B, Takeshita K, Cheng DM, Matthew Volm, Wasserheit C, Demopoulos R, Chanan-Khan A. Effect of highly active antiretroviral therapy on survival in patients with AIDS associated Kaposi's sarcoma treated with chemotherapy. J Clin Oncol 2001; 19: 3848-3851.

10. Bower M, Fox P. Fife K, Gill J, Nelson M, Gazzard B. Highly active antiretroviral therapy prolongs time to treatment failure in Kaposi's sarcoma. AIDS 1999; 13 : 2105-2111

11. Gomez-Sucerquia LJ, Blas-Garcia A, Marti-Cabrera M, Esplugues JV, Apostolova N. Profile of stress and toxicity gene expression in human hepatic cells treated with Efavirenz. Antiviral Res 2012; 94: 232-241.

12. Ware JA, Simons M, Eds. Angiogenesis in health and disease: Basic mechanisms and clinical applications. New York: Marcel Dekker; 2000. pp 47-73.

13. Hagedorn M, Balke $M$, Schmidt A, Bloch W, Kurz $H$, Javerzat S, Rousseau B, Wilting J, Bikfalvi A. VEGF coordinates interaction of pericytes and endothelial cells during vasculogenesis and experimental angiogenesis. Dev Dyn 2004; 230(1): 23-33

14. Nishida $N$, Yano $H$, Nishida $T$, Kamura $T$, Kojiro $M$. Angiogenesis in Cancer. Vasc Health Risk Manag 2006; 2(3): 213-219.
15. McBride WG. Thalidomide and congenital abnormalities. Lancet 1961; 2: 1358

16. Lenz W. Thalidomide and Congenital Anomalies. Lancet 1962; 1: 45

17. D'Amato RJ, Loughnan MS, Flynn E, Folkman J. Thalidomide is an inhibitor of angiogenesis (fibroblast growth factor/rabbit cornea). Proc Natl Acad Sci USA 1994; 91(9): 4082-4085.

18. Folkman J. Tumor angiogenesis: therapeutic implications. N Engl J Med 1971; 285(21): 1182-1186.

19. Sampaio E, Sarno E, Galill R, Cohn Z, Kaplan G. Thalidomide selectively inhibits tumor necrosis factor alpha production by stimulated human monocytes. J Exp Med 1991; 173(3): 699-703.

20. Yoshida S, Ono M, Shono T, Izumi H, Ishibashi T, Suzuki $H$, Kuwano M. Involvement of interleukin-8, vascular endothelial growth factor, and basic fibroblast growth factor in tumor necrosis factor alpha-dependent angiogenesis. Mol. Cell Biol 1997; 17(7): 4015-4023.

21. Wang J1, Zhang G, Bambara RA, Li D, Liang $H, W u H$, Smith HM, Lowe NR, Demeter LM, Dykes C. Nonnucleoside Reverse Transcriptase Inhibitor-Resistant HIV Is Stimulated by Efavirenz during Early Stages of Infection. J Virol 2011; 85(20): 10861-10873.

22. van Leth $F$, Phanuphak $P$, Ruxrungtham $K$, Baraldi $E$, Miller S, Gazzard B, Cahn P, Lallo UG, van der Westhuizen IP, Malan DR et al. Comparison of first-line antiretroviral therapy with regimens including nevirapine, Efavirenz, or both drugs, plus stavudine and lamivudine: a randomised open-label trial, the 2NN Study. Lancet 2004; 363 (9417): 1253-1263.

23. NIH publication no. 85-23, revised 1985. Principles of Laboratory Animal Care. Available from: https://grants.nih.gov/grants/olaw/guide-for-the-careand-use-of-laboratory-animals.pdf.

24. Hamburger V, Hamilton HL. A series of normal stages in the development of the chick embryo. 1951. Dev. Dyn 1992; 195(4): 231-272.

25. Weisz A, Koren B, Cohen $T$, Neufeld G, Kleinberger $T$, Lewis BS, Flugelman MY. Increased Vascular Endothelial Growth Factor 165 Binding to Kinase Insert Domain-Containing Receptor After Infection of Human Endothelial Cells by Recombinant Adenovirus Encoding the Vegf165 Gene: Basic Sci Reports. Circulation 2001; 103(14): 1887-1892.

26. Neben K, Moehler T, Benner A, Kraemer A, Egerer G, Ho $A D$, Goldschmidt $H$. Dose-dependent Effect of Thalidomide on Overall Survival in Relapsed Multiple Myeloma. Clin Cancer Res November 2002; 8: $3377-$ 3382.

27. Dvorak HF. Vascular permeability factor/vascular endothelial growth factor: a critical cytokine in tumor angiogenesis and a potential target for diagnosis and therapy. J Clin Oncol 2002; 20(21):4368-4380.

28. Drake CJ. Embryonic and adult vasculogenesis. Birth Defects Res C Embryo Today 2003; 69(1): 73-82.

29. Senger DR, Galli SJ, Dvorak AM, Perruzzi CA, Harvey VS, Dvorak HF. Tumor cells secrete a vascular Trop J Pharm Res, November 2017; 16(11): 2746 
McNeil et al

permeability factor that promotes accumulation of ascites fluid. Science 1983; 219: 983-985.

30. Zhang ZG, Zhang L, Jiang $Q$, Zhang R, Davies $K$, Powers C, van Bruggen N, Chopp M.VEGF enhances angiogenesis and promotes blood-brain barrier leakage in the ischemic brain. J Clin nvest. 2000; 106(7): 829838. 